\title{
Advances at TRIUMF-ISAC and decay of neutron-rich Cd studied with GRIFFIN
}

\author{
P.E. Garrett ${ }^{1,2, \star}$, N. Bernier ${ }^{3,4}$, R. Dunlop ${ }^{1}$, A.D. MacLean ${ }^{1}$, V. Bildstein ${ }^{1}$, I. Dillmann ${ }^{3}, A$. \\ Jungclaus $^{5}$, C.E. Svensson ${ }^{1}$, C. Andreoiu ${ }^{6}$, G.C. Ball ${ }^{3}$, H. Bidaman ${ }^{1}$, P. Boubel ${ }^{1}$, C. Burbadge ${ }^{1}$, \\ R. Caballero-Folch ${ }^{3}$, M.R. Dunlop ${ }^{1}$, L.J. Evitts ${ }^{1}$, F. Garcia ${ }^{6}$, A.B. Garnsworthy, G. Hackman ${ }^{3}$, \\ S. Hallam ${ }^{3,7}$, J. Henderson ${ }^{3}$, S. Ilyushkin ${ }^{8}$, D. Kisliuk ${ }^{1}$, R. Krücken ${ }^{3,4}$, J. Lassen $^{3,9}$, R. Li ${ }^{3}$, \\ E. MacConnachie ${ }^{3}$, E. McGee ${ }^{1}$, M. Moukaddam ${ }^{3}$, B. Olaizola ${ }^{3}, K$. Ortner ${ }^{6}$, E. Padilla-Rodal ${ }^{10}$, \\ J. Park ${ }^{3,4}$, O. Paetkau ${ }^{3}$, C.M. Petrache ${ }^{11}$, J.L. Pore ${ }^{6}$, A.J. Radich ${ }^{1}$, P. Ruotsalainen ${ }^{3}$, J. \\ Smallcombe ${ }^{3}$, J.K. Smith ${ }^{3}$, S.L. Tabor ${ }^{12}$, A. Teigelhöfer ${ }^{3,9}$, J. Turko ${ }^{1}$, K. Whitmore ${ }^{6}$, and T. Zidar ${ }^{1}$ \\ ${ }^{1}$ Department of Physics, University of Guelph, Guelph, ON N1G2W1 Canada \\ ${ }^{2}$ Department of Physics and Astronomy, University of the Western Cape, Belleville 7441, South Africa \\ ${ }^{3}$ TRIUMF, 4004 Wesbrook Mall, Vancouver, British Columbia V6T 2A3, Canada \\ ${ }^{4}$ Department of Physics and Astronomy, University of British Columbia, Vancouver, British Columbia \\ V6T1Z4, Canada \\ ${ }^{5}$ Instituto de Estructura de la Materia, CSIC, E-28006 Madrid, Spain \\ ${ }^{6}$ Department of Chemistry, Simon Fraser University, Burnaby, British Columbia V5A1S6, Canada \\ ${ }^{7}$ Department of Physics, University of Surrey, Guildford GU2 7XH, United Kingdom \\ ${ }^{8}$ Department of Physics, Colorado School of Mines, Golden, Colorado 80401, USA \\ ${ }^{9}$ Department of Physics and Astronomy, University of Manitoba, Winnipeg, Manitoba R3T2N2, Canada \\ ${ }^{10}$ Universidad Nacional Autónoma de México, Instituto de Ciencias Nucleares, AP 70-543, México City \\ 04510, DF, México \\ ${ }^{11}$ Centre de Sciences Nucléaires et Sciences de la Matière, CNRS/IN2P3, Université Paris-Saclay, 91405 \\ Orsay, France \\ ${ }^{12}$ Department of Physics, Florida State University, Tallahassee, Florida 32306, USA
}

\begin{abstract}
.
The $\beta$-decay half lives of nuclei near the $r$-process path are critical information required for abundance calculations, especially those near neutron number $N=82$. Specifically, the nuclei below doubly-magic ${ }^{132} \mathrm{Sn}$ are key, and play an important role in the formation and shape of the second $r$-process abundance peak. The half lives in this region are challenging to measure due to the significant $\beta$-delayed neutron decay branches and the population of isomeric states with half lives comparable to the ground states. However, by measuring the time distribution of $\gamma$ rays, these complications can be eliminated. This requires, however, a very efficient $\gamma$-ray spectrometer since the production of isotopes in this region is very limited. The new GRIFFIN array at TRIUMF-ISAC provides the high efficiency required for these measurements.

Recent improvements in the quality of the beams produced at TRIUMF, employing the IG-LIS device, are outlined, as well as the current status of the ARIEL facility. The GRIFFIN spectrometer and its use are briefly described. The experiment to measure the half lives of ${ }^{128-130} \mathrm{Cd}$ is outlined and the results given, and some examples of the power
\end{abstract}

^e-mail: pgarrett@physics.uoguelph.ca 
of GRIFFIN to expand decay schemes, specifically for the decay of ${ }^{128} \mathrm{Cd}$ to ${ }^{128} \mathrm{In}$, are given.

\section{Introduction}

Advancements in the production, separation, and re-acceleration of unstable nuclei has propelled nuclear physics into a new era. While much remains to be done in the study and understanding of nuclei along the line of stability, many of the leading questions in nuclear science can only be addressed through the direct study of isotopes far from stability. Many laboratories around the world are now dedicated to the development and use of unstable isotopes. In this contribution, we focus on the Isotope Separator And Accelerator (ISAC) at TRIUMF - an Isotope Separator On-Line (ISOL) facility that produces some of the most intense radioactive beams in the world. A new development at TRIUMF, the Advanced Rare IsotopE Laboratory (ARIEL), is currently under construction with completion and full implementation of its beams scheduled in 2022. One of the flagship facilities that makes use of the beams from ISAC, and also those of the future ARIEL, is the Gamma-Ray Infrastructure For Fundamental Investigations of Nuclei (GRIFFIN). This new spectrometer offers unprecedented sensitivity for $\beta$-decay measurements of radioactive nuclei that are produced in copious amounts, typically in the region of stable nuclei, as well as those produced at very low intensities at the extremes of existence.

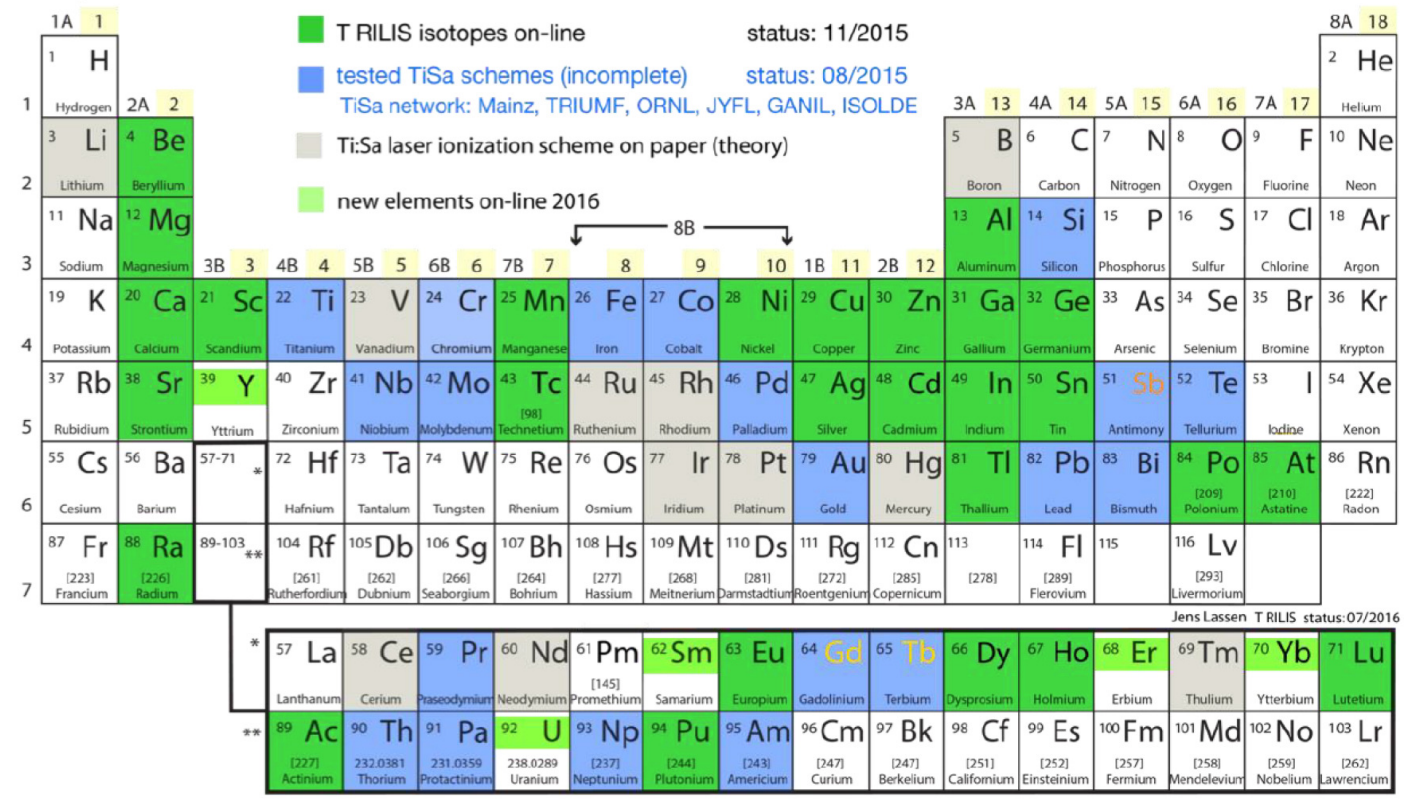

Figure 1. Status of species ionised with laser-ion source TRILIS at TRIUMF-ISAC. Species highlighted in green have been extracted and used in experiments, those in blue have ionisation schemes tested offline, whereas those in grey have theoretical schemes but not yet tested. 


\section{TRIUMF-ISAC and ARIEL}

\subsection{ISAC}

The TRIUMF-ISAC facility was developed through the late 1990's and 2000's, with the first beams available in 1998 [1]. ISAC is based on the ISOL technique with the 520-MeV TRIUMF main cyclotron, that can deliver up to $100 \mu \mathrm{A}$ of protons to ISAC, acting as the primary driver. The targets available [2] to date include $\mathrm{SiC}, \mathrm{TiC}, \mathrm{NiO}, \mathrm{Nb}, \mathrm{ZrC}$, Ta, $\mathrm{ThC}_{x}$, and $\mathrm{UC}_{x}$ that can be operated with a variety of ion sources; a Re Surface ion source, a FEBIAD (Forced Electron Beam Induced Arc Discharge) source, and TRILIS (TRIumf Laser Ion Source) [3], and the IG-LIS (Ion Guide - Laser Ion Source) [4]. The thickness of the targets varies, but typically are on the order of $20 \mathrm{~g} / \mathrm{cm}^{2}$. The impinging proton beam induces (mainly) spallation and fission reactions on the target nuclei; the products of the reaction then diffuse and effuse from the material where they can be ionized. In order to facilitate the diffusion, the targets are heated to a temperature of $\approx 2300^{\circ} \mathrm{C}$ [2]. Following ionisation, the ions are guided to the mass separator that has a resolution of $M / \Delta M \approx 2000$ [3], and then via beam transport lines to the various experimental facilities. Some notable extracted yields include ${ }^{26} \mathrm{Al}$ with $>10^{10}$ pps, ${ }^{37,38} \mathrm{~K}$ with $>10^{9} \mathrm{pps}$, and ${ }^{211-213} \mathrm{Fr}$ with $>10^{9} \mathrm{pps}$.

TRILIS has become one of the main ion sources used at ISAC, and was used for the experiments described below. It uses all solid state lasers, operating with tunable pulsed titanium-sapphire lasers pumped by a Nd:YAG laser. Figure 1 shows the elements as of 2016 for which laser-ionised beams had been produced (in green) or the schemes had been developed and tested offline (blue). Use of the laser ionisation schemes enables selectivity of isomers provided that the magnitude of the hyperfine effect can overcome the combined bandwidth of the lasers and the Doppler broadening. For some species, the magnetic moments for ground states and isomers are sufficiently large to enable a hyperfine splitting to allow separation; ${ }^{115} \mathrm{Ag}$ presented such as case. Shown in Fig. 2 are the yields for the ground state of ${ }^{115} \mathrm{Ag}$ (scale on the right, fit to the yields in red) and ${ }^{115} \mathrm{Ag}^{m}$ (scale on the left, fit to the yields in blue). As can be seen, outstanding separation of the isomer and ground state can be achieved through tuning the laser taking into account the hyperfine structure.

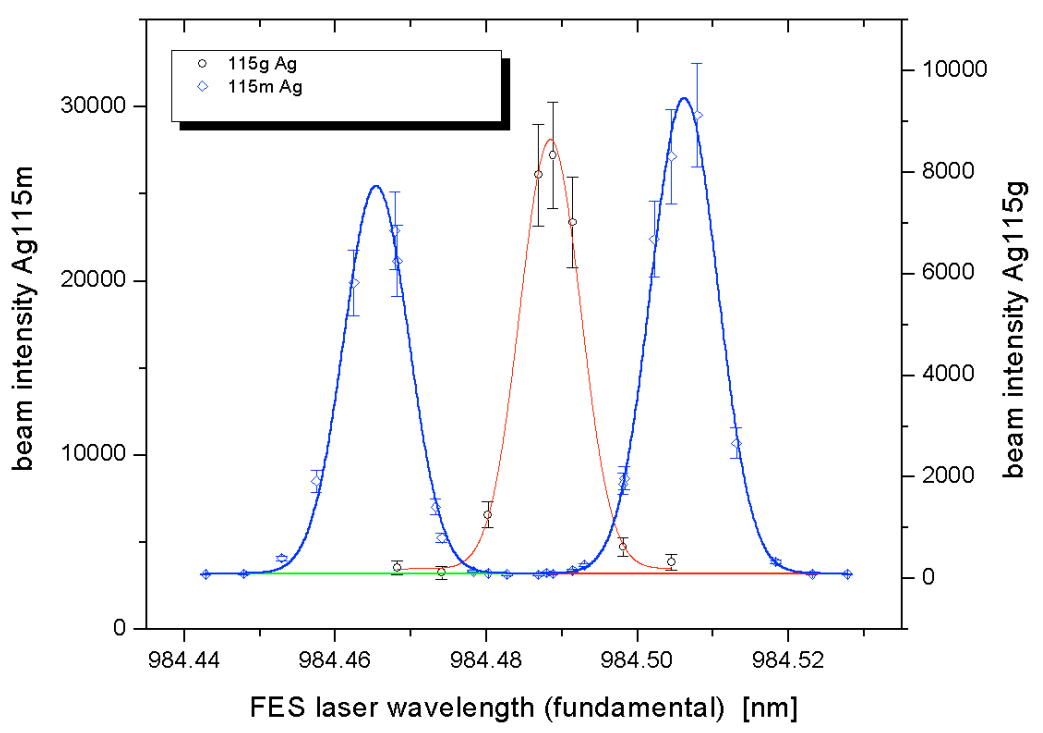

Figure 2. Demonstration of the ability of the laser ionisation source to separate isomer and ground state of ${ }^{115} \mathrm{Ag}$. The yields are shown in units of ions/s for the ground state (right) and isomeric state (left). 


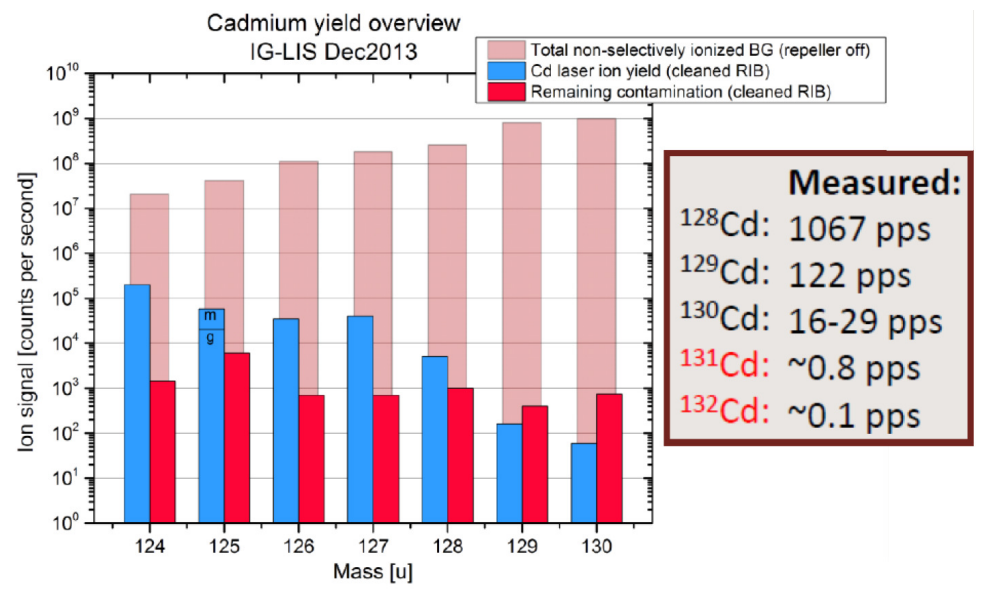

Figure 3. Results of yield measurements with IG-LIS. The lightred bars indicate the total yield of a particular mass measured with IG-LIS in full transmission mode (without the use of repeller electrode) that allows surface ionised species to be transferred. The blue bars are the measured laserionised yields of the $\mathrm{Cd}$ isotope with IG-LIS operation in surfacesuppression mode, with the red bars the yields of all remaining contamination.

While the laser ionisation itself is extremely selective, since the target is heated to a high temperature surface ionisation can occur and it can be rather efficient for some species. A recent development to overcome the potentially large beam backgrounds that could be present is the IG-LIS. IG-LIS is designed to suppress surface ionised species by employing a repeller plate electrode that creates an electrostatic potential barrier to the positively-charged ions, only allowing neutral species to propagate into the transfer tube, which is kept cold (relative to the high temperatures of the target itself) where they can be ionised by the lasers, and extracted. Figure 3 shows the yields of $\mathrm{Cd} v s$. other ionised species measured without the use of IG-LIS and those with IG-LIS in operation. While the use of IG-LIS causes a decrease in the extracted yield of Cd species of interest by a factor of 20-50, it suppresses the surface-ionised products by a factor of $\approx 10^{6}$, leading to a greatly enhanced signal-to-noise ratio.

\subsection{ARIEL}

TRIUMF-ISAC, like many radioactive-beam laboratories worldwide, has a significant oversubscription of its facilities, and consequently has a substantial backlog of experiments. In order to address the needs of the community, construction began on ARIEL in 2010, with phase-I completion in 2014. ARIEL includes a new 35-MeV electron LINAC that will deliver $100 \mathrm{~kW}$ of beam power on its targets, as well as a new, second proton beam line also capable of delivery of $100 \mu \mathrm{A}$ of $500 \mathrm{MeV}$ protons. Phase-II of ARIEL is currently underway, and together with the CANREB project (which constitutes phase-III) that will provide a high-resolution $M / \Delta M \approx 20000$ mass separator and a new EBIS-based (Electron Beam Ion Source) charge-state breeder, will broaden the scope and quality of beams to be delivered to the ISAC experimental halls. Phase-II is currently scheduled for completion in the 2022 time frame, although the CANREB components will be available in 2020. The phase-IV of the ARIEL project will see the development of multi-user capability, with the provision of delivery of 3 radioactive beams to the low-energy and high-energy halls simultaneously, with approved experiments scheduled in 2023. Figure 4 shows a schematic of the new facility.

\section{GRIFFIN}

A wide-ranging program of nuclear structure and fundamental symmetries investigations utilizing $\beta$ decay was conducted with the $8 \pi$ spectrometer [5-7], arguably the most powerful device of its time 


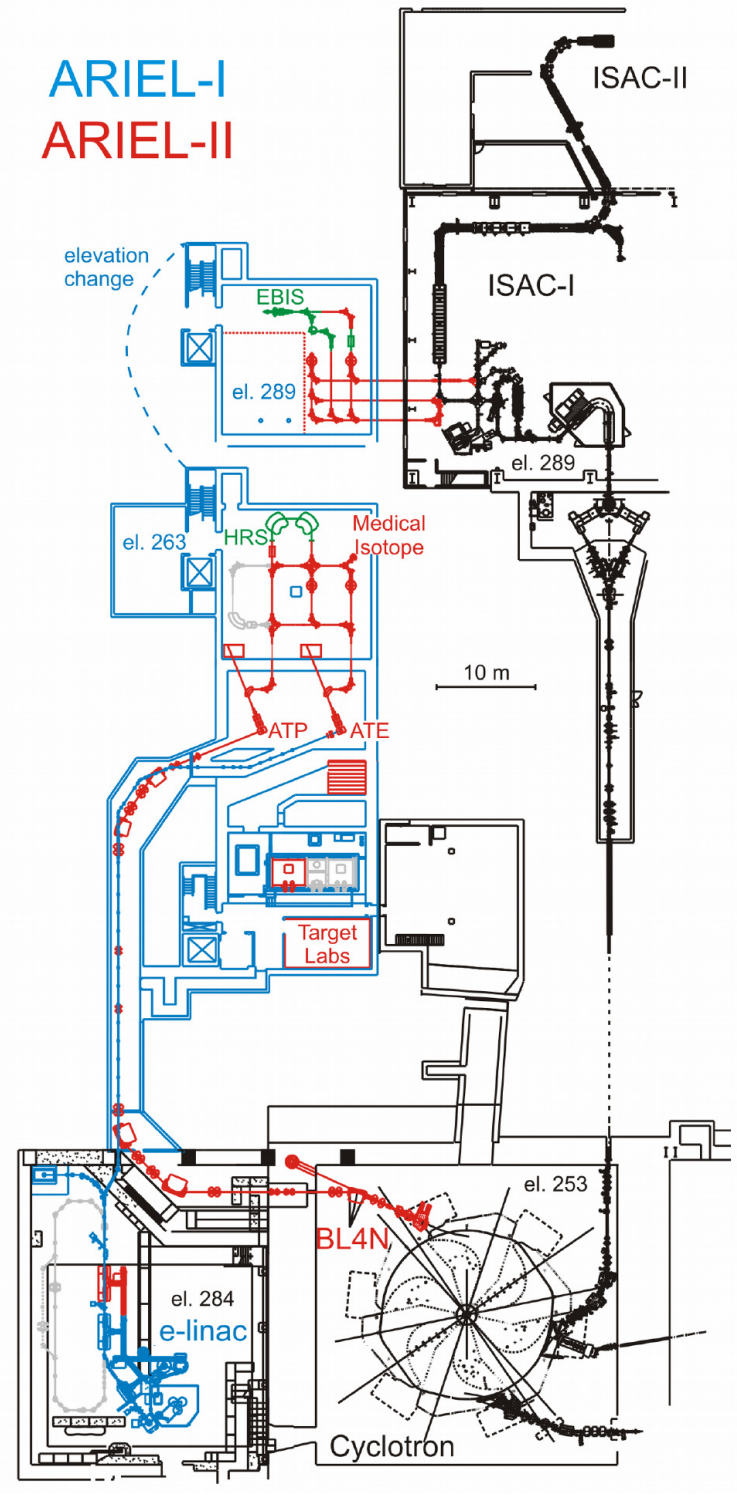

Figure 4. Schematic of the layout of the ARIEL facility. The infrastructure outlined in black is the existing cyclotron (bottom) and ISAC facility (top). The phase-I of the ARIEL project, highlighted in blue, included all of the civil construction required for ARIEL, as well as the main components of the $35-\mathrm{MeV}$ electron LINAC, located in the hall adjacent to the main cyclotron. The new infrastructure for ARIEL-II is shown in red, and includes a new proton beam line (BL4N), target stations for both the electron and new proton beam lines, and beam transport systems for delivery to multiple users. ARIEL-II is tightly coupled to the CANREB project (shown in green) that will provide a new high-resolution mass separator capable of $M / \Delta M \approx 20000$, and a new chargebreeding system based on an EBIS with a RFQ cooler/buncher, and Nier spectrometer.

dedicated to such studies. However, in order to push the limits of sensitivity and ability to perform studies with beams having rates below $1 \mathrm{pps}$, a new spectrometer, GRIFFIN, has been constructed [8]. Figure 5 shows a photograph of the device in its current configuration. GRIFFIN is comprised of up to 16 large-volume high-purity germanium detectors (HPGe) detectors, with each Ge crystal averaging $41 \%$ relative efficiency [9], arranged in a rhombicuboctahedron. The triangular openings of the rhombicuboctahedron can house different detectors, such as smaller, single crystal Ge detectors, DESCANT detectors [10], or, much more commonly, an array of $\mathrm{LaBr}_{3}(\mathrm{Ce})$ detectors. GRIFFIN utilizes the same set of ancillary systems as the previous $8 \pi$ spectrometer; the Scintillating ElectronPositron Tagging Array (SCEPTAR) - an array of $20 \times 1.6 \mathrm{~mm}$ thick plastic scintillators for $\beta$-particle detection; the Pentagonal Array of Conversion Electron Spectrometers (PACES) - an array of $5 \times 5 \mathrm{~mm}$ 


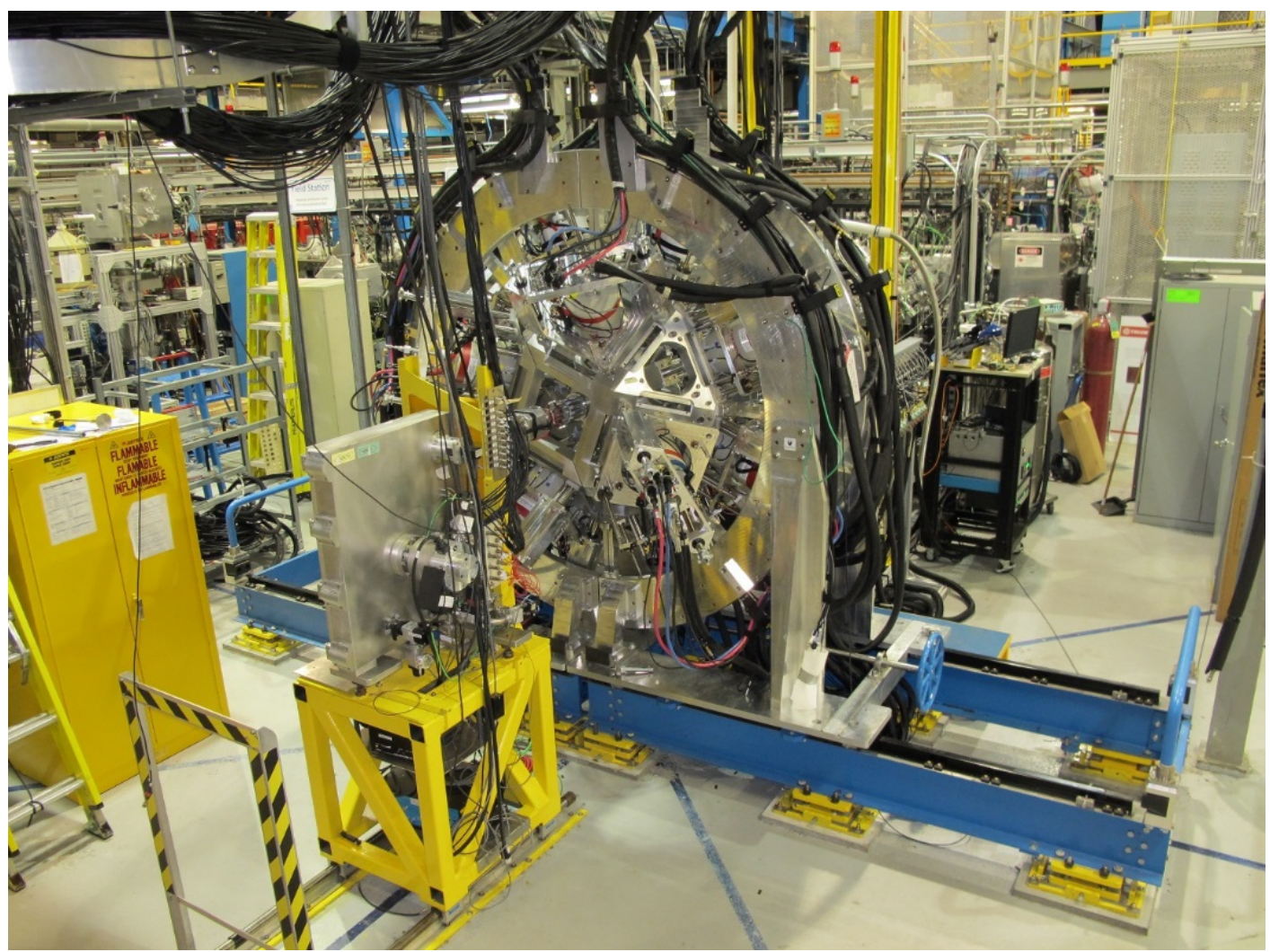

Figure 5. Photograph of the GRIFFIN array at TRIUMF-ISAC. In the foreground is the box of the Moving Tape Collector. The array consists of up to 16 large-volume HPGe clover detectors, and uses the SCEPTAR and PACES ancillary devices from the $8 \pi$ spectrometer [5]. Up to 8 additional detectors, such as $\mathrm{LaBr}_{3}$, can be placed in the triangular openings (one of which is visible in the upper right-hand side of the array). The array was designed to accommodate BGO anti-Compton and background suppression shields for the $\mathrm{HPGe}$ and $\mathrm{LaBr}_{3}(\mathrm{Ce})$ detectors that are currently being acquired.

thick high-resolution $\mathrm{Si}(\mathrm{Li})$ detectors; and the Moving Tape Collector (MTC) - a device that consists of a continuous loop of tape on which beam can be deposited at the centre of the array and then transported outside of the focal volume once the counting period is completed. These ancillary detectors are described more fully in Ref. [5].

The improvements in sensitivity of GRIFFIN over the $8 \pi$ are shown in Ref. [8]. At $1 \mathrm{MeV}$, when using the clover detectors in add-back mode, the absolute photopeak efficiency is $19 \%$. For $\gamma-\gamma$ coincidences, this represents a factor of $\approx 300$ greater than the $8 \pi$ spectrometer. In order to take full advantage of this tremendous increase in efficiency, a new fully digital data acquisition system (DAQ) has been developed [12], designed to enable high-precision measurements required for tests of fundamental symmetries, such as those for the superallowed Fermi $\beta^{+}$emitters, as well as very high throughput for nuclear structure studies near stability. The DAQ uses the MIDAS data acquisition system that interfaces with three levels of hardware; the GRIF-16 modules that digitize the detector signals (14-bit $100 \mathrm{MHz}$ sampling ADCs), a GRIF-C slave module that collect the data from the 

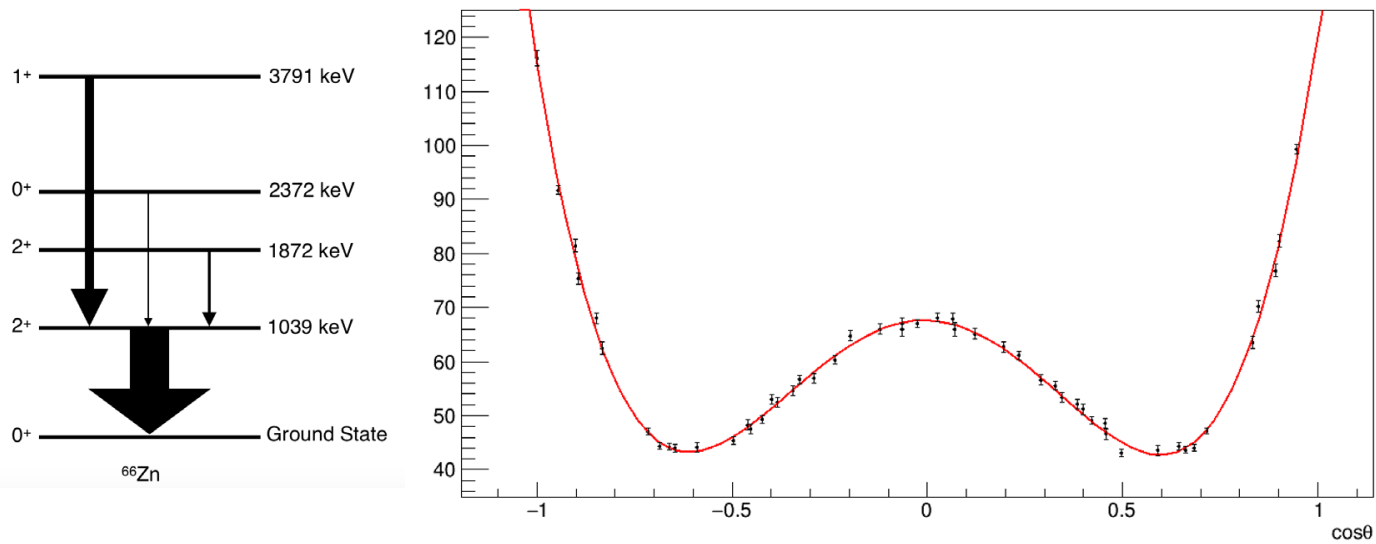

Figure 6. Example of $\gamma-\gamma$ angular correlations with the GRIFFIN spectrometer. On the left is shown a partial decay scheme of ${ }^{66} \mathrm{Zn}$ observed in the decay of ${ }^{66} \mathrm{Ga}$. The data on the right are the coincidence intensities (in arbitrary units) observed for the $0^{+} \rightarrow 2^{+} \rightarrow 0^{+}$cascade $2372-\mathrm{keV} \rightarrow 1039-\mathrm{keV} \rightarrow 0-\mathrm{keV}$ cascade as a function of $\cos \theta$ where $\theta$ is the angular difference between the individual Ge crystals. The red curve is not a fit to the data (apart from an overall normalisation), but the results of GEANT4 simulations of the cascade.

GRIF-16 modules, and a GRIF-C Master module that collects and filters the data from the lower GRIF-C slaves. A clock signal at $50 \mathrm{MHz}$ is distributed to all modules from a single GRIF-Clk module, and slow control of external devices, such as the MTC or control of the beam on/off, is performed through a programmable GRIF-PPG module that outputs NIM or TTL level signals. The GRIFFIN DAQ is extensively described in Ref. [12]. The ultimate design goal is to process data from the HPGe detectors with each crystal counting at $50 \mathrm{kHz}$, and write data to disk as a sustained rate of approximately $300 \mathrm{MB} / \mathrm{s}$. Rates as high as $100 \mathrm{MB} / \mathrm{s}$ have already been achieved.

One of the main advantages of the high efficiency of GRIFFIN is the ability to perform $\gamma-\gamma$ angular correlations. Shown in Fig. 6 are data from ${ }^{66} \mathrm{Ga}$ decay to ${ }^{66} \mathrm{Zn}$. The data shown in right panel are the coincidence intensities, as a function of the cosine of the angular difference between the individual Ge crystals, for the $0^{+} \rightarrow 2^{+} \rightarrow 0^{+}$cascade shown in the level scheme of the left panel. The curve on the plot is a simulated curve for the cascade performed with the GEANT4 code; only an overall normalisation has been fit to the data.
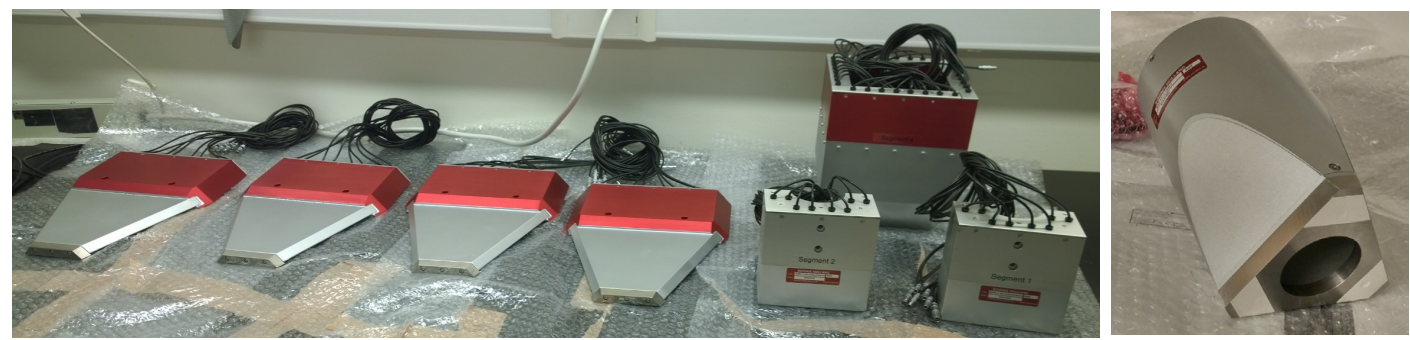

Figure 7. Photographs of an HPGe BGO anti-Compton and background suppression shield set (left), and a shield designed for use with the $\mathrm{LaBr}_{3}(\mathrm{Ce})$ detectors (right). 
The next phase of GRIFFIN, which will see the array fully equipped with BGO anti-Compton and background suppression shields, is currently being implemented. The HPGe detectors will be equipped with side, front, and back shields, very similar to those of the TIGRESS array [13] and shown in Fig. 7. In addition, eight BGO shields are being acquired for use with the ancillary $\mathrm{LaBr}_{3}(\mathrm{Ce})$ detectors, and one such shield is also shown in Fig. 7. The implementation of the antiCompton and background suppression shields into GRIFFIN will be completed by autumn 2018, and will, for example, greatly enhance the sensitivity for detection of weak, low-energy $\gamma$-rays, and reduce the systematic uncertainties in lifetime measurements performed with the $\mathrm{LaBr}_{3}(\mathrm{Ce})$ detectors.

\section{Decay of neutron-rich In and Cd}

The combination of IG-LIS and GRIFFIN for measurements of exotic and weakly produced beams is aptly demonstrated by a recent experiment to study the decays of neutron-rich nuclei $Z=48,49$ nuclei near $N=82$. A $9.8 \mu \mathrm{A} 500-\mathrm{MeV}$ proton beam bombarded a $\mathrm{UC}_{x}$ target; for this experiment IG-LIS was vital as the isotopes of interest, namely ${ }^{128-130} \mathrm{Cd}$, would not have been detectable if the copious surface-ionised species remained unsuppressed. One of the main goals of the experiment was a measurement of the lifetimes of ${ }^{128-130} \mathrm{Cd}$, since there were discrepant results for ${ }^{129,130} \mathrm{Cd}$ [14-16]. The lifetimes of these nuclei are important for $r$-process nucleosynthesis calculations, and especially the experimental lifetime of ${ }^{130} \mathrm{Cd}$ that was used to adjust the quenching factor of the Gamow-Teller operator used in shell model calculations for this entire region of isotopes. The power of GRIFFIN with its extraordinary efficiency that enabled the measurement of $\beta-\gamma$ coincidences; by
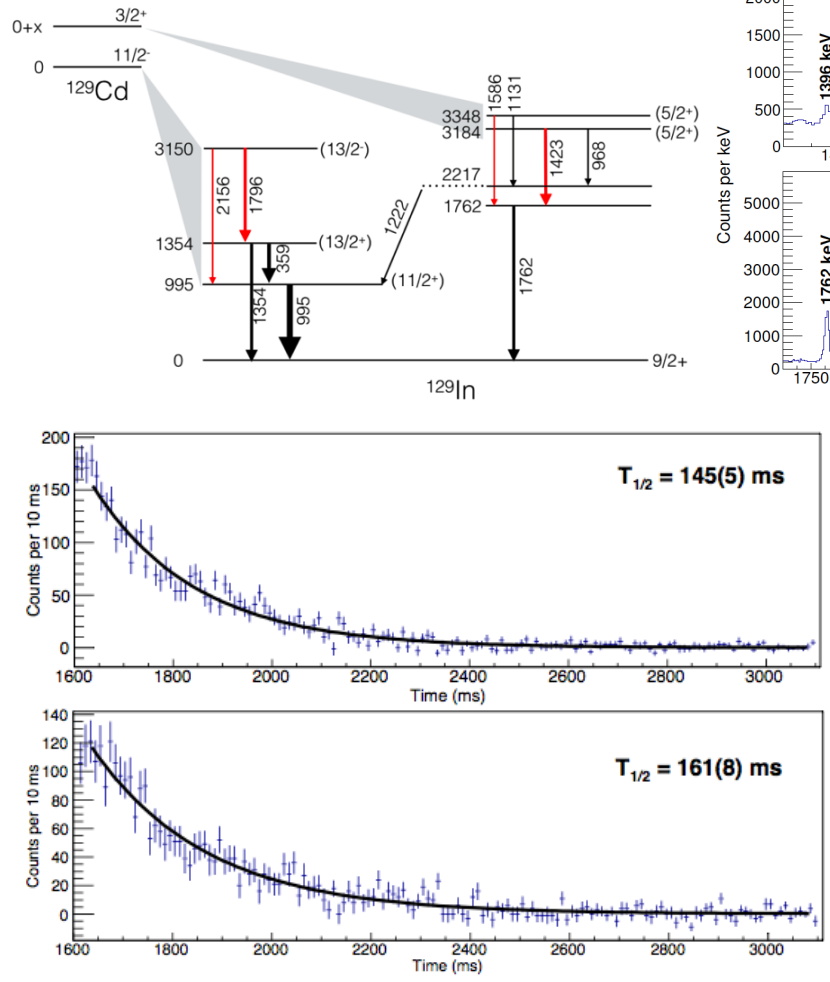

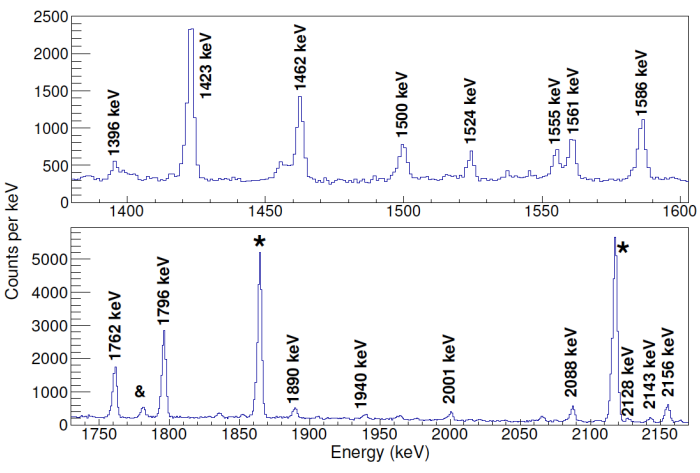

Figure 8. The partial decay scheme of ${ }^{129} \mathrm{Cd}$ (top left), showing the principle $\gamma$ rays associated with the ground state and isomeric state decay. The transitions in red are those on which coincidence gates were placed to extract the $\beta$-particle decay curves. The top right panel shows the partial $\gamma$-ray spectrum in coincidence with $\beta$ particles detected in SCEPTAR, displaying the excellent statistics achieved. The bottom panel are the resulting $\beta$-particle decay curves for the ground state (top) and isomeric state (bottom) after the $\gamma$ ray coincidence conditions were placed [16]. 


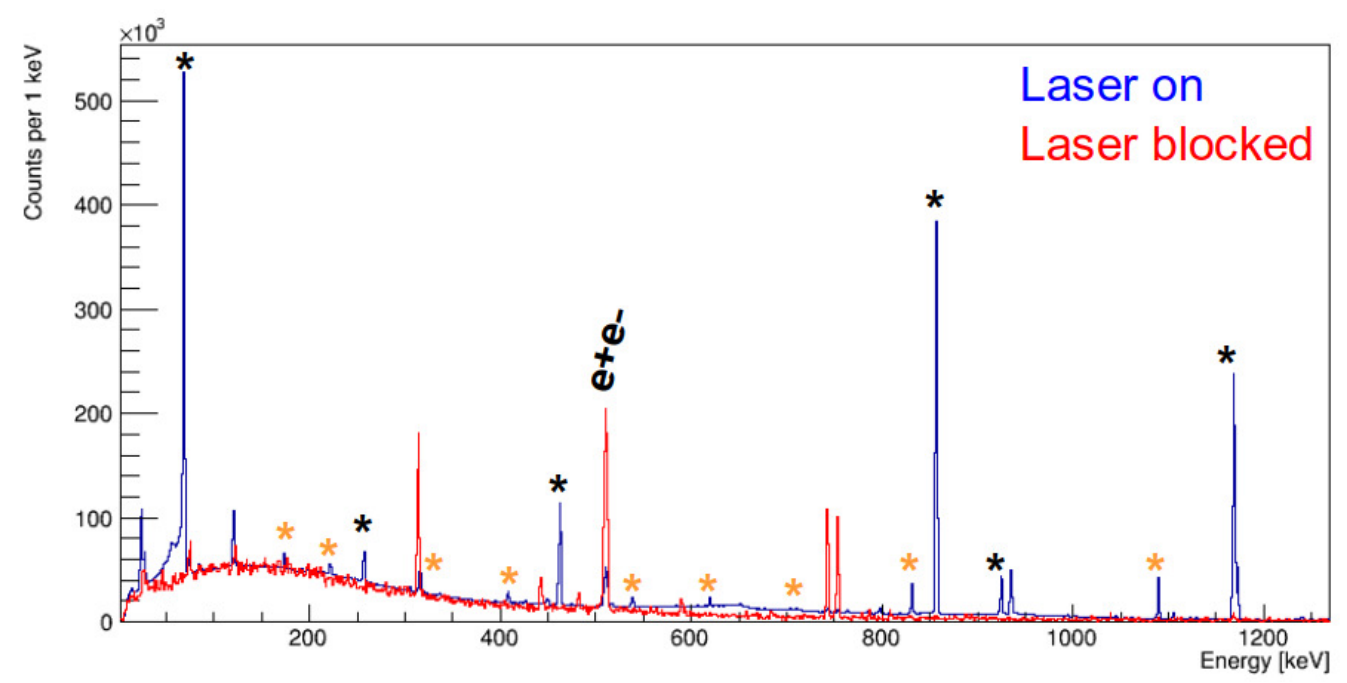

Figure 9. Spectrum observed for decay of the $A=128$ beam delivered to the GRIFFIN spectrometer. The spectrum in blue corresponds to the decay $\gamma$-rays observed with the ionisation lasers on, whereas that in red is the spectrum observed with the lasers blocked from reaching the IG-LIS. The peaks with a black asterisk correspond to known ${ }^{128} \mathrm{Cd}$ decay $\gamma$ rays, whereas those with an orange asterisk are identified as newly observed ${ }^{128} \mathrm{Cd}$ decay lines [17].

selecting specific, well-resolved and unique $\gamma$ rays with the HPGe detectors, the $\beta$-decay lifetime could be measured by performing fits to the decay curve of the coincident $\beta$ particles detected with SCEPTAR. An example of this is shown in Fig. 8 [16]. The top-left panel displays a partial decay scheme of ${ }^{129} \mathrm{Cd}$, and shows that coincidence gating on the $995-\mathrm{keV}$ transition, while this may isolate the decay of ${ }^{129} \mathrm{Cd}$, would not resolve the decays of the ground state from those of the isomeric state. Coincidence gates on the 1796- and $2156-\mathrm{keV} \gamma$-ray transitions select only those $\beta$ particles that result from the decay of the $11 / 2^{-129} \mathrm{Cd}$ ground state, whereas coincidence gates on the 1423 - and $1586-\mathrm{keV}$ $\gamma$ rays select $\beta$ particles resulting from the decay of the $3 / 2^{+}$isomeric state. The resulting $\beta$-particle decay curves are shown in the bottom panel. The extracted lifetimes from the measurements [16] were $246.2 \pm 2.1 \mathrm{~ms}$ for ${ }^{128} \mathrm{Cd}, 158 \pm 8$ and $148 \pm 3 \mathrm{~ms}$ for the $3 / 2^{+}$and $11 / 2^{-}$states of ${ }^{129} \mathrm{Cd}$, and $126 \pm 4 \mathrm{~ms}$ for the decay of ${ }^{130} \mathrm{Cd}$, in agreement with those of Ref. [15]. Further details of the present results can be found in Ref. [16].

In addition to the goal of measuring the half lives, the excellent sensitivity of GRIFFIN has enabled the level schemes of the daughters observed in the decay to be expanded. For example, Fig. 9 displays portions of the $\gamma$-ray spectra observed in coincidence with $\beta$ particles for the delivered $A=128$ beam [17]. The use of the laser on/blocked comparison enables quick determination of the parentage of the observed $\gamma$ rays. (Note that the laser-blocked spectrum has been scaled to the smooth background of the laser-on spectrum.) A number of newly observed transitions assigned to the decay of ${ }^{128} \mathrm{Cd}$ are marked with an orange asterisk [17]; those with a black asterisk were previously known.

The real power of GRIFFIN becomes evident when seeking to establish $\gamma-\gamma$ coincidence relations with beam intensities of 1000 ions/s or less. Figure 10 shows $\gamma$-ray coincidence spectra for the ${ }^{128} \mathrm{Cd}$ decay data; the beam rate was approximately 1000 ions/s, and data were collected for 7 hours. New transitions, corresponding to those identified in Fig. 9, are readily identified, and the coincidence relations will enable their placement in the decay scheme. This part of the analysis is ongoing [17], 

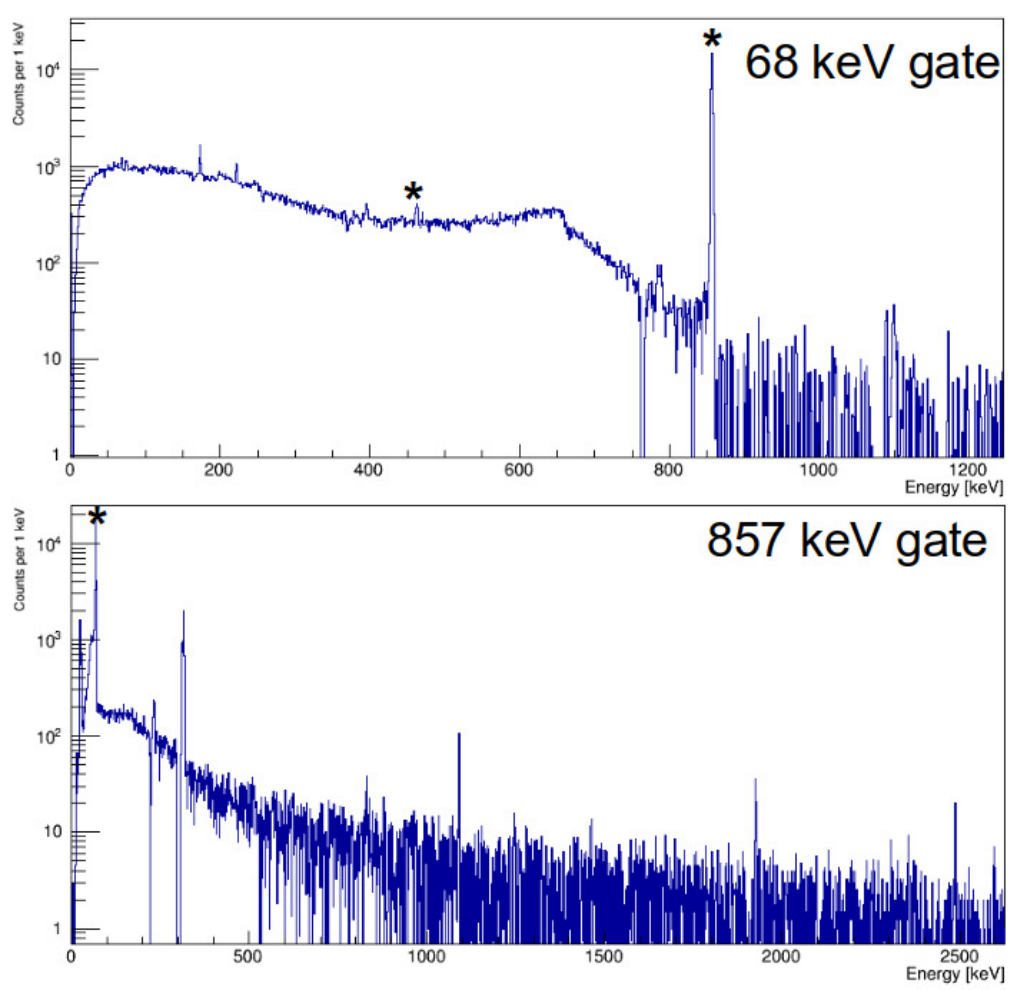

Figure 10. Examples of $\gamma$-coincidence spectra observed [17] with the GRIFFIN spectrometer for decay of ${ }^{128} \mathrm{Cd}$. The top panel shows the coincidences with the $68-\mathrm{keV}$ $315-\mathrm{keV} \rightarrow 247-\mathrm{keV}$ transition in ${ }^{128} \mathrm{In}$, with the bottom panel the coincident $\gamma$ rays with the $857-\mathrm{keV}$ $1172-\mathrm{keV} \rightarrow 315-\mathrm{keV}$ transition. The peaks with a black asterisk correspond to known coincident $\gamma$ rays in ${ }^{128} \mathrm{In}$.

and together with a $\gamma-\gamma$ angular correlation analysis, offer the possibility to firmly establish spins and parities for some of the excited states in ${ }^{128} \mathrm{In}$.

\section{Summary}

$\beta$-decay spectroscopy is entering a new era at the TRIUMF facility. With the advances in ion-source technologies that enable higher-purity radioactive beams, to the ARIEL facility that will result in dramatic increases in both intensities of certain species and time available for experiments, TRIUMF will remain at the forefront of nuclear science for years to come. GRIFFIN, a new $\gamma$-ray spectrometer dedicated to $\beta$-decay studies, is well-poised to take advantage of the opportunities provided by the TRIUMF facility. The first result from GRIFFIN, measuring accurately and precisely the lifetimes for the $N=80,81$, and 82 isotopes of $\mathrm{Cd}$, is an apt demonstration of the capabilities of this coupling. To date (as of March 2017), in 2.5 years of operation, more than 27 different radioactive beams have been delivered to GRIFFIN spanning nuclear structure, nuclear astrophysics, and experiments related to tests of fundamental symmetries, with many more to come.

\section{References}

[1] J. Dilling, R. Krücken, and G. Ball, Hyper. Int. 225, 1 (2014).

[2] M. Dombsky and P. Kunz, Hyper. Int. 225, 17 (2014).

[3] P.G. Bricault, F. Ames, M. Dombsky, P. Kunz, and J. Lassen, Hyper. Int. 225, 25 (2014). 
[4] S. Raeder et al., Rev. Sci. Inst. 85, 033309 (2014).

[5] P.E. Garrett, A.J. Radich, J.M. Allmond, C. Andreoiu, G.C. Ball, P.C. Bender, L. Bianco, V. Bildstein, H. Bidaman, R. Braid, C. Burbadge, S. Chagnon-Lessard, D.S. Cross, G. Deng, G.A Demand, A. Diaz Varela, M.R. Dunlop, R. Dunlop, P. Finlay, A.B. Garnsworthy, G.F. Grinyer, G. Hackman, B. Hadinia, S. Ilyushkin, B. Jigmeddorj, D. Kisliuk, K. Kuhn, A.T. Laffoley, K.G. Leach, A.D. MacLean, J. Michetti-Wilson, D. Miller, W. Moore, B. Olaizola, J.N. Orce, C.J. Pearson, J.L. Pore, M.M. Rajabali, E.T. Rand, F. Sarazin, J.K. Smith, K. Starosta, C.S. Sumithrarachchi, C.E. Svensson, S. Triambak, J. Turko, Z.M. Wang, J.L. Wood, J. Wong, S.J. Williams, S.W. Yates, E.F. Zganjar, J. Phys. G. Conf. Ser. 639, 012006 (2015).

[6] A.B. Garnsworthy and P.E. Garrett, Hyper. Int. 225, 121 (2014).

[7] A.B. Garnsworthy, E. Phys. J. Web of Conf. 93, 01032 (2015).

[8] C.E. Svensson and A.B. Garnsworthy, Hyper. Int. 225, 127 (2014).

[9] U. Rizwan, A.B. Garnsworthy, C. Andreoiu, G.C. Ball, A. Chester, T. Domingo, R. Dunlop, G. Hackman, E.T. Rand, J.K. Smith, K. Starosta, C.E. Svensson, P. Voss, J. Williams, Nucl. Instrum. Meth. A820, 126 (2016).

[10] P.E. Garrett, Hyper. Int. 225, 137 (2014).

[11] V. Bildstein, P.E. Garrett, S.F. Ashley, G.C. Ball, L. Bianco, D. Bandyopadhyay, J. Bangay, B.P. Crider, G. Demand, G. Deng, I. Dillmann, A. Finlay, A.B. Garnsworthy, G. Hackman, B. Hadinia, R. Krücken, K.G. Leach, J-P. Martin, M.T. McEllistrem, C.J. Pearson, E.E. Peters, F.M. PradosEstévez, A. Radich, F. Sarazin, C. Sumithrarachchi, C.E. Svensson, J.R. Vanhoy, J. Wong, and S.W. Yates, E. Phys. J. Web of Conf. 93, 07005 (2015).

[12] A.B. Garnsworthy, C.J. Pearson, D. Bishop, B. Shaw, J.K. Smith, M. Bowry, V. Bildstein, G. Hackman, P.E. Garrett, Y. Linn, J.-P. Martin, W.J. Mills, C.E. Svensson, Nucl. Instrum. Meth. A853, 85 (2017).

[13] G. Hackman and C.E. Svensson, Hyper. Int. 225, 241 (2014).

[14] B. Fogelberg et al., Proc. Intern. Conf. Nuclear Data for Science and Technology, Mito, Japan (Saikon Publishing Co., Tokyo, Japan, 1988), p. 837.

[15] G. Lorusso, S. Nishimura, Z.Y. Xu, A. Jungclaus, Y. Shimizu, G.S. Simpson, P.-A. Söderström, H. Watanabe, F. Browne, P. Doornenbal, G. Gey, H.S. Jung, B. Meyer, T. Sumikama, J. Taprogge, Zs. Vajta, J. Wu, H. Baba, G. Benzoni, K.Y. Chae, F.L. Crespi, N. Fukuda, R. Gernhäuser, N. Inabe, T. Isobe, T. Kajino, D. Kameda, G.D. Kim, Y.-K. Kim, I. Kojouharov, F.G. Kondev, T. Kubo, N. Kurz, Y.K. Kwon, G.J. Lane, Z. Li, A. Montaner-Pizá, K. Moschner, F. Naqvi, M. Niikura, H. Nishibata, A. Odahara, R. Orlandi, Z. Patel, Zs. Podolyák, H. Sakurai, H. Schaffner, P. Schury, S. Shibagaki, K. Steiger, H. Suzuki, H. Takeda, A. Wendt, A. Yagi, and K. Yoshinaga, Phys. Rev. Lett. 114, 192501 (2015).

[16] R. Dunlop, V. Bildstein, I. Dillmann, A. Jungclaus, C.E. Svensson, C. Andreoiu, G.C. Ball, N. Bernier, H. Bidaman, P. Boubel, C. Burbadge, R. Caballero-Folch, M.R. Dunlop, L.J. Evitts, F. Garcia, A.B. Garnsworthy, P.E. Garrett, G. Hackman, S. Hallam, J. Henderson, S. Ilyushkin, D. Kisliuk, R. Krücken, J. Lassen, R. Li, E. MacConnachie, A. D. MacLean, E. McGee, M. Moukaddam, B. Olaizola, E. Padilla-Rodal, J. Park, O. Paetkau, C.M. Petrache, J.L. Pore, A.J. Radich, P. Ruotsalainen, J. Smallcombe, J.K. Smith, S.L. Tabor, A. Teigelhöfer, J. Turko, and T. Zidar, Phys. Rev. C 93, 062801(R) (2016).

[17] N. Bernier et al., unpublished. 